\title{
Rapid analysis of spatial distribution of PVPP and hardness of Yinhuang dispersible tablets by NIR-CI
}

\author{
Yifei Wang, Zhisheng $\mathrm{Wu}^{*, \S}$, Zhaoyi Wang, Manfei Xu, Xinyuan Shi \\ and Yanjiang Qiao ${ }^{\dagger}, \S$ \\ Beijing University of Chinese Medicine, P. R. China, 100102 \\ Key Laboratory of TCM-information Engineering \\ of State Administration of TCM \\ Beijing 100102, P. R. China \\ *wzs@bucm.edu.cn \\ †yjqiao@263.net
}

Received 5 September 2014

Accepted 30 October 2014

Published 2 December 2014

\begin{abstract}
The present study aimed at investigating the relationship between tablet hardness and homogeneity of different Yinhuang dispersible tablets by near-infrared chemical imaging (NIR-CI) technology. The regularity of best hardness was founded between tablet hardness and the spatial distribution uniformity of Yinhuang dispersible tablets. The ingredients homogeneity of Yinhuang dispersible tablets could be spatially determined using basic analysis of correlation between analysis (BACRA) method and binary image. Then different hardnesses of Yinhuang dispersible tablets were measured. Finally, the regularity between tablet hardness and the spatial distribution uniformity of Yinhuang dispersible tablets was illuminated by quantifying the agglomerate of polyvinyl poly pyrrolidone (PVPP). The result demonstrated that the distribution of PVPP was unstable when the hardness was too large or too small, while the agglomerate of PVPP was smaller and more stable when the best tablet hardness was $75 \mathrm{~N}$. This paper provided a novel methodology for selecting the best hardness in the tabletting process of Chinese Medicine Tablet.
\end{abstract}

Keywords: Near-infrared chemical imaging; Yinhuang dispersible tablets; PVPP; tablet hardness.

\section{Introduction}

Chinese Medicine Tablet (CMT) is the most commonly used dosage form among modern traditional Chinese medicine pharmaceutics. Tablet hardness is a critical pharmaceutical process parameters in quality control. Tabletting forces have a deep influence on ingredient spatial distribution. ${ }^{1}$ Ingredient spatial distribution information is closely related to the quality attribute of tablet because spatial distribution is directly related to the bioavailability. ${ }^{2-7}$

$\S_{\text {Corresponding authors. }}$

This is an Open Access article published by World Scientific Publishing Company. It is distributed under the terms of the Creative Commons Attribution 3.0 (CC-BY) License. Further distribution of this work is permitted, provided the original work is properly cited. 
Nowadays, wet analysis, mass spectrometry (MS) or high-performance liquid chromatography (HPLC), etc., which were used to evaluate the tablet homogeneity depends on different contents of active pharmaceutical ingredients in different tablets. Anil and Pravin reviewed novel techniques for solubility, dissolution rate and bioavailability enhancement of class II and IV drugs because enhancement of solubility, dissolution rate and bioavailability is a very challenging task in drug development. ${ }^{8}$ Huang et al. investigated the relationship between the dissolution rate and bioavailability of paracetamol, in which the result showed a good linear relationship. ${ }^{9}$

However, wet analysis cannot provide information about spatial distribution of active pharmaceutical ingredients in tablets. Furthermore, more attention should be paid to the spatial distribution of active pharmaceutical ingredients because complex compounds distribution has a critical impact on the dissolution rate and therapeutic performance of CMT.

Recently, near infrared chemical imaging (NIR$\mathrm{CI}$ ) is a visual technique in analytical technology. NIR-CI adds a dimension about spatial distribution to the spectral information. ${ }^{10}$ Roggo et al. measured the effect on dissolution properties of pilot-scale produced tablets using NIR-CI. The result showed that hydrophobic magnesium stearate protected the tablet cores from moisture and therefore slowed dissolution. ${ }^{11}$ Saravanan et al. investigated the effect of the concentration of Eudragit L100, microcrystalline cellulose and tablet hardness on in vitro release of cephalexin extended release tablet. The dissolution results showed that a higher amount of Eudragit in tablet composition and higher tablet hardness resulted in reduced drug release. ${ }^{12}$

Furthermore, increasing the complexity of ingredients in traditional Chinese medicine pharmaceutics requires new methods for rapid and direct visualization of the spatial distribution of active pharmaceutical ingredient (API) in CMT. Wu et al. have firstly studied API distribution and homogeneity of CMT by NIR-CI. ${ }^{13,14} \mathrm{Wu}$ et al. demonstrated a novel application of NIR-CI for monitoring the blending process of Yinhuang powder. The result illuminated MBMRSTDEV could be a useful method for visualizing the trend of the blending process using NIR-CI. ${ }^{15}$

However, no paper has reported the influence of tablet hardness on spatial distribution of ingredients in CMT. Traditional technology measured the hardness of tablets to meet the quality requirements, which ignored the influence of tablet hardness on pharmaceutical ingredients distribution. Simultaneously, the measurement of tablet hardness is destructive and off-line, which cannot timely obtain process quality information of the intermediate, thus lead to the quality unstability of tablets.

The spatial distribution and homogeneity with different hardness in Yinhuang dispersible tablets was investigated by NIR-CI technology, as well as the relationship between tablet hardness and spatial distribution uniformity of Yinhuang dispersible tablets. In this paper, this research focused on the relationship between spatial distribution of polyvinyl poly pyrrolidone (PVPP) and tabletting forces. PVPP distribution has a critical impact on the tabletting forces, dissolution rate and therapeutic performance of Yinhuang dispersible tablets. Therefore, the best hardness was investigated according to the characteristics of PVPP in CMT in pilot-scale production process.

\section{Materials and Methods}

\subsection{Materials}

Extracts of Radix Scutellariae Baicalensis and Flos Lonicerae were provided by Key Laboratory of TCM-information Engineering of State Administration of TCM (Beijing, China). PVPP was purchased from Yunhong Chemical Industry Corporation (Shanghai, China). Microcrystalline cellulose (101QD, International special products, USA) and magnesium stearate were purchased from Zhanwang Excipients Corporation (Zhejiang, China).

\subsection{Preparation of Yinhuang dispersible tablets}

The formulation of Yinhuang dispersible tablet $(0.3 \mathrm{~g} \text { per tablet })^{16}$ included Radix S. Baicalensis extract (100 g), F. Lonicerae extract (40 g), PVPP (21 g), Microcrystalline cellulose (15.4 g) and Magnesium stearate $(0.7 \mathrm{~g})$. A uniform dry powder mixture of active ingredients and excipients was obtained by mixing in a mortar according to the prescription's proportion after sifting with 100 mesh (0.15mm).

Yinhuang dispersible tablets were produced by direct powder compression technique performed by 
pilot-scale ZPS10 rotary tablet machine (Xinyuan pharmaceutical machinery, Inc., Shanghai, China). A total of 15 samples with hardness between 10 and $200 \mathrm{~N}$ were produced by adjusting process parameters of rotary tablet machine. Each pure compound reference tablet was also produced to obtain pure spectrum during NIR-CI scanning.

\subsection{NIR-CI equipment and software}

Yinhuang dispersible tablet was measured directly on a NIR line mapping system (Spectrum Spotlight 400 FT-IR and 400 N FT-NIR Imaging Systems, PerkinElmer, UK) using a microscope slide. For each acquisition, 16 spectra were collected from a linear mercury cadmium telluride (MCT) detector array. Each spectrum of Yinhuang dispersible tablet was the average of 16 scans, each of which used the wavenumber region 7800 $4000 \mathrm{~cm}^{-1}$, pixel size $25 \times 25 \mu \mathrm{m}$ and a spectral resolution of $16 \mathrm{~cm}^{-1} \cdot{ }^{13,17}$ After sample scanning, the tablet hardness was tested by Tablet Hardness Tester (Kraemer Elektronik, Inc., Germany).

\subsection{Data treatment}

The Spotlight 400 software (Spectrum Spotlight 400 FTIR and $400 \mathrm{~N}$ FT-NIR Imaging Systems, PerkinElmer, UK) was utilized to acquire the data. Hyper View (PerkinElmer), Matlab version 7.0 (MathWorks Inc., USA) and Image-Pro Plus software (Media Cybernetics, USA) were used for data processing. Some of the algorithms used in this paper were developed in Matlab platform by ourselves.

\section{Results and Discussions}

\subsection{Raw NIR images of Yinhuang dis- persible tablets of different hardness}

Figure 1 showed raw NIR images of Yinhuang dispersible tablets of different hardness. As shown in Fig. 1, raw images could reflect different hardness message of Yinhuang dispersible tablets according to signal characteristic in the region of $4000-7800 \mathrm{~cm}^{-1}$. In addition, five pure spectra were extracted from each pure image, respectively (Data was not shown). As a consequence, the hardness of 15 samples was measured, as shown in Fig. 1.

\subsection{Identification by basic analysis of the correlation between analysis method}

\subsubsection{Characteristic analysis of five pure spectra}

NIR image was preprocessed by different methods, such as the combination of normalize and second derivative, smoothing average with a window size of 9 points, the combination of smoothing average with a window size of 9 points and normalize. As reported in our previous research, ${ }^{13-15}$ the combination of smoothing average with a window size of 9 points and normalize provided the best pretreatment results.

Therefore, to clarify the differences between two pure spectra, based on the best pretreatment methods, correlation image was calculated by the basic analysis of correlation between analysis (BACRA) method, as shown in the formula.

$$
r=\frac{\sum X_{i} Y_{i}-\frac{\sum X_{i} \sum Y_{i}}{N}}{\sqrt{\left(\sum X_{i}^{2}-\frac{\left(\sum X_{i}\right)^{2}}{N}\right)\left(\sum Y_{i}^{2}-\frac{\left(\sum Y_{i}\right)^{2}}{N}\right)}},
$$

where $X_{i}$ is the spectrum of sample image; $Y_{i}$ is each pure spectrum of the ingredient in Yinhuang dispersible tablets (e.g., $i=1,2,3 \ldots m$ ); $N$ is the number of spectral variables.

The results were shown in Fig. 2. There were high correlation coefficients $(r)$ between $F$. Lonicerae extract and microcrystalline cellulose. It indicated that the tiny difference between $F$. Lonicerae extract and microcrystalline cellulose could not be identified using the BACRA method. On the contrary, the spectra of other three pure components in Yinhuang dispersible tablets could be identified by BACRA method.

\subsubsection{Determination of PVPP distribution in Yinhuang dispersible tablets by BACRA method}

The correlation-coefficient images of Radix S. Baicalensis extract, PVPP, magnesium stearate and Yinhuang dispersible tablets were investigated. The correlation coefficients between the pure spectrum of Radix S. Baicalensis extract and all the absorbance spectra of the Yinhuang dispersible tablets were $0.76-0.84$. In addition, the correlation 


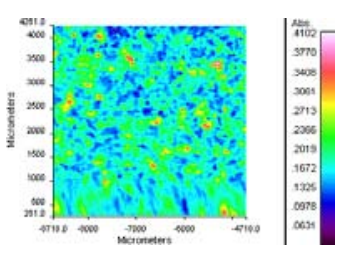

$1-1(16 \mathrm{~N})$

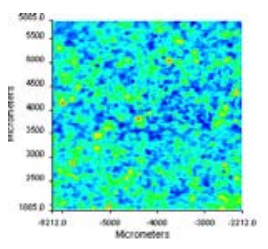

$1-6(75 N)$

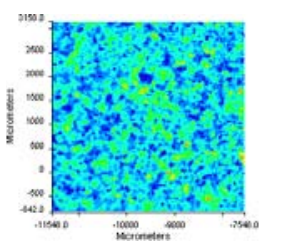

$1-11(155 \mathrm{~N})$

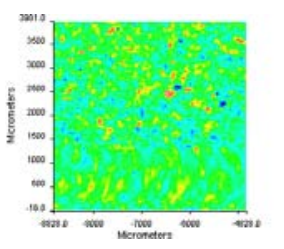

$1-2(24 N)$
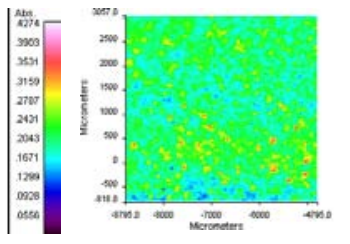

$1-7(103 N)$
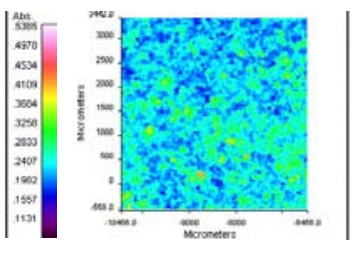

$1-12(158 \mathrm{~N})$

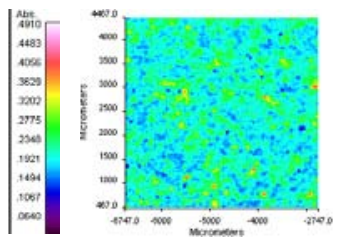

$1-3(47 N)$

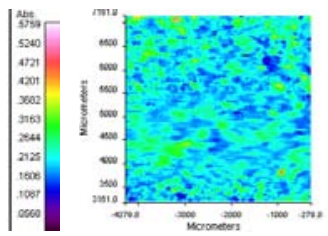

$1-8(111 N)$
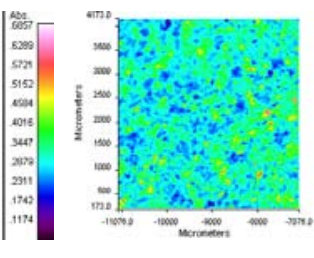

$1-13(159 \mathrm{~N})$
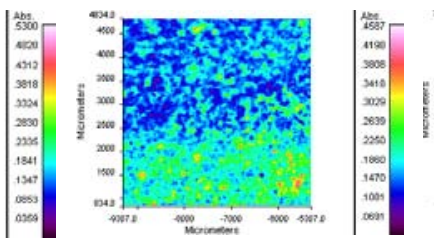

$1-4(52 N)$

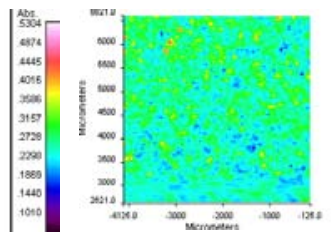

$1-9(117 N)$

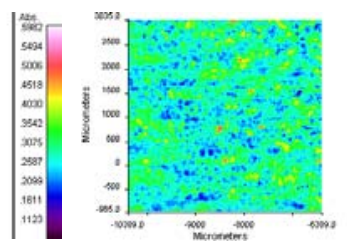

$1-14(180 \mathrm{~N})$

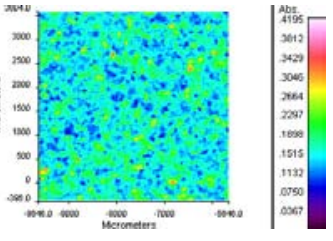

$1-5(75 N)$

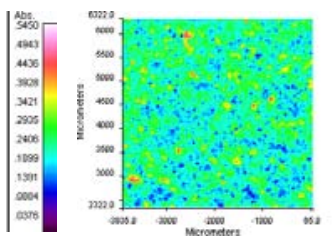

$1-10(135 \mathrm{~N})$
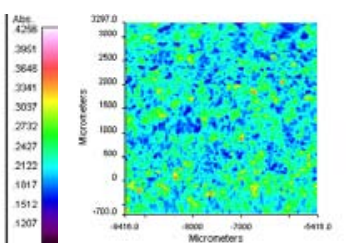

$1-15(200 N)$

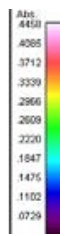

Fig. 1. Raw NIR images of Yinhuang dispersible tablets of different hardness.
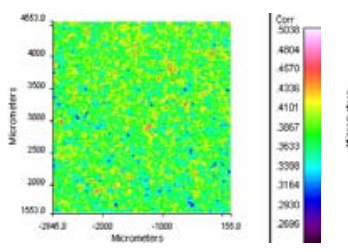

2-1

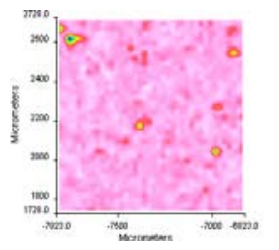

2-6

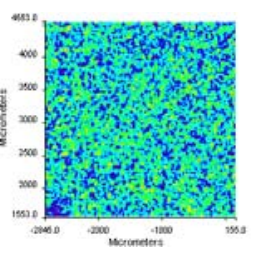

2-2

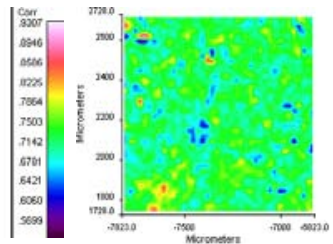

2-7

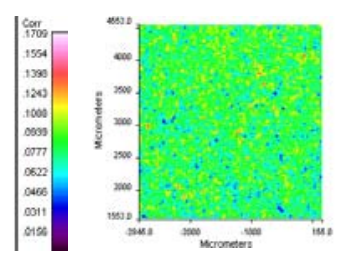

$2-3$

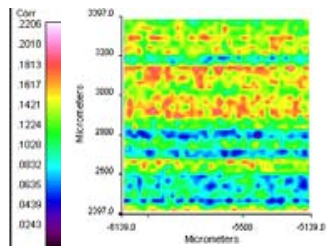

2-8
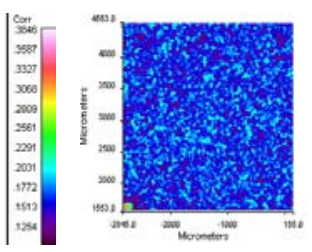

$2-4$
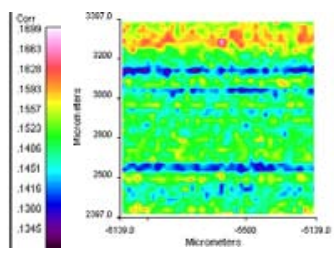

2-9
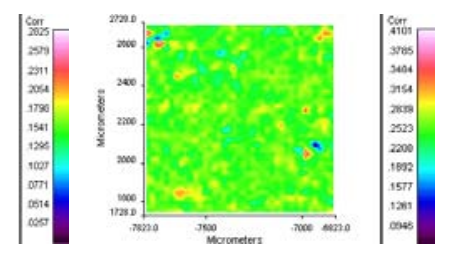

$2-5$

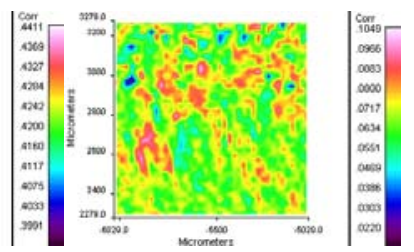

2-10

Fig. 2. Characteristic analysis of five pure spectra. 2-1, the correlation image from Radix S. Baicalensis extract with pure F. Lonicerae extract image; 2-2, the correlation image from Radix S. Baicalensis extract with pure PVPP image; 2-3, the correlation image from Radix S. Baicalensis extract with microcrystalline cellulose image; 2-4, the correlation image from Radix S. Baicalensis extract with magnesium stearate image; 2-5, the correlation image from $F$. Lonicerae extract with PVPP image; 2-6, the correlation image from $F$. Lonicerae extract with microcrystalline cellulose image; 2-7, the correlation image from $F$. Lonicerae extract with magnesium stearate image; 2-8, the correlation image from PVPP with microcrystalline cellulose image; 2-9, the correlation image from PVPP with magnesium stearate image; 2-10, the correlation image from microcrystalline cellulose with magnesium stearate image. 


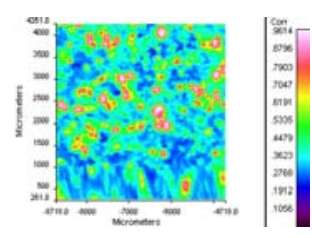

3-1

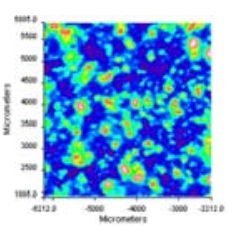

3-6

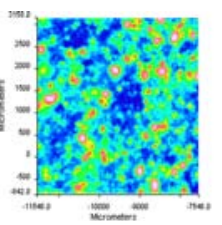

3-11

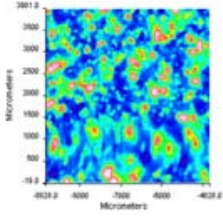

3-2
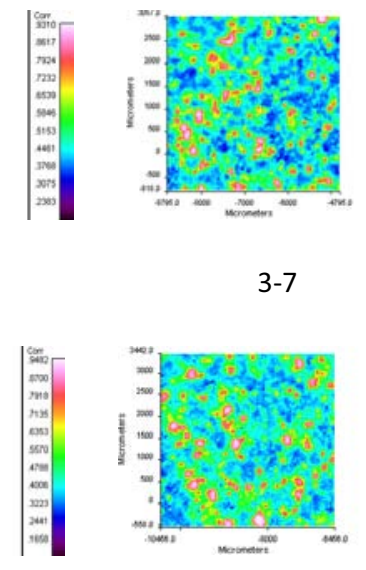

3-12

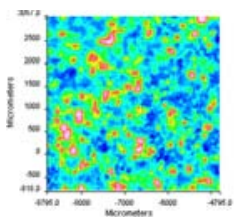

3-7
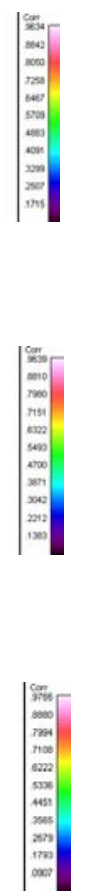

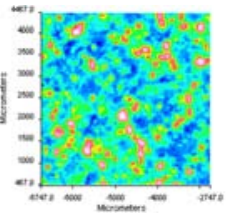

3-3

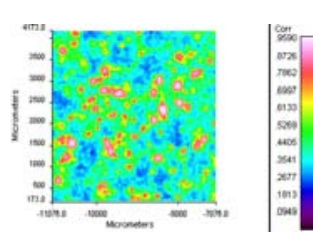

3-13
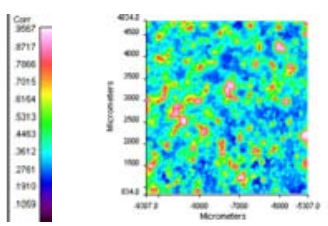

3-4

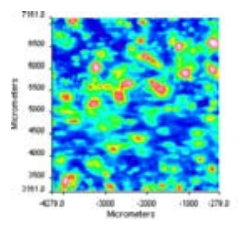

3-8

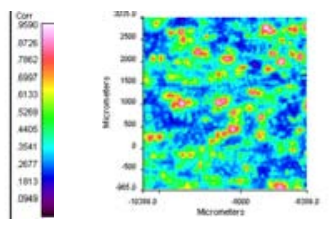

3-14

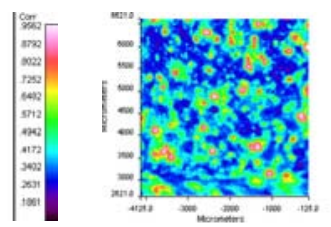

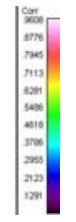
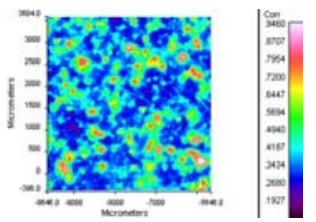

3-5
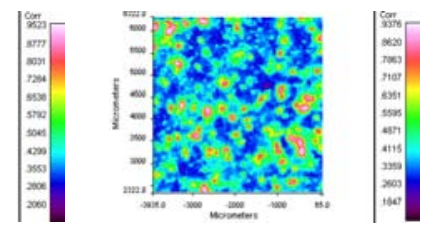

$3-10$
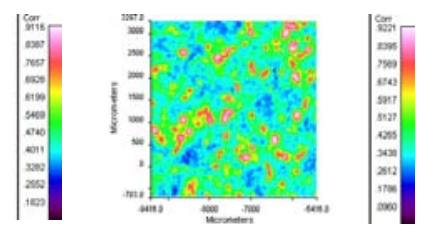

3-15

Fig. 3. BACRA analysis of PVPP with Radix S. Baicalensis extract, magnesium stearate image in Yinhuang dispersible tablets.

coefficients between the pure spectrum of magnesium stearate and all the spectra of Yinhuang dispersible tablets were below 0.5. Therefore, Radix $S$. Baicalensis extract and magnesium stearate could not be identified by BACRA methodology.

However, as shown in Fig. 3, the correlation coefficients between the pure spectrum of PVPP and all the absorbance spectra of the Yinhuang dispersible tablets were all above 0.91, which indicated the existence of PVPP. PVPP zones were distinguished by correlation-coefficient from highest to lowest in the correlation coefficient maps, which illustrated the spatial distribution of PVPP in Yinhuang dispersible tablets.

\subsection{Homogeneity analysis of PVPP spatial distribution}

The result was further depicted by binary image, as shown in Fig. 4. The aggregation of PVPP in each sample was visualized in binary image. The comparison of each sample could be easily illuminated.

The spatial distribution of PVPP was clearly identified by binary images. However, for a technique used in quality control, the data should be objective. Therefore, a certain parameter to evaluate the degree of agglomeration was adopted to quantify the agglomeration of each image. The relationship between the aggregation quantitative parameter of PVPP and tablet hardness was clarified by the data statistics, as shown in Table 1. The aggregation size, average aggregation area and average aggregation length of PVPP is shown among different hardness of Yinhuang dispersible tablets.

Firstly, the average agglomeration area of PVPP gets large and fluctuant in the unstable period, in which the value of average agglomeration area is above 3000 pixels. The tablet hardness range of this unstable period is $16-52 \mathrm{~N}$ and $158-200 \mathrm{~N}$. In addition, the average agglomeration length of PVPP exceeds 70 pixels. Secondly, agglomerate number of PVPP is relatively few and stable within the tablet hardness of $75 \mathrm{~N}$.

Thus, tablet hardness should be controlled within a certain small range to ensure the homogeneity of PVPP distribution in compression process. The result validated that the best tablet hardness range was $75 \mathrm{~N}$ according to the variance trend. It provides the methodology about the measurement of critical process parameters (CPP), which affect critical quality attributes in the CMT tablet process, such as hardness. 


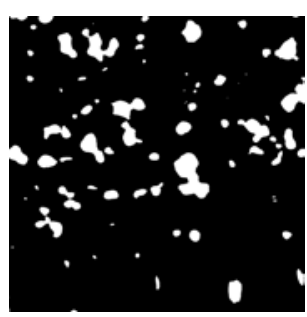

3-1

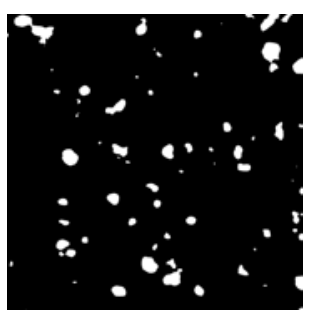

$3-6$

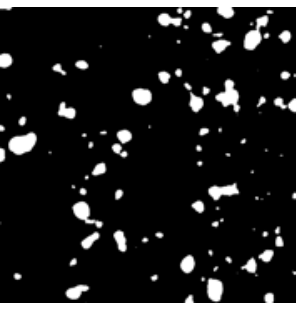

3-11

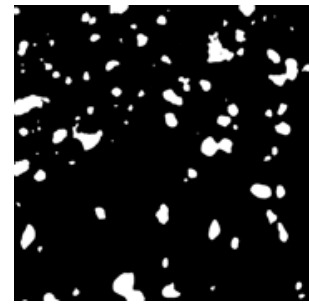

3-2

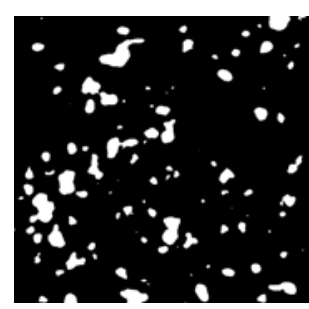

3-7

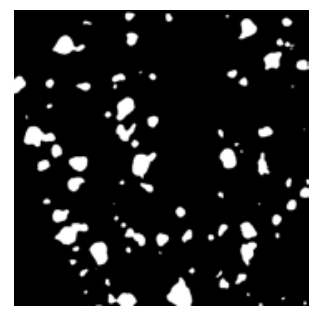

3-12

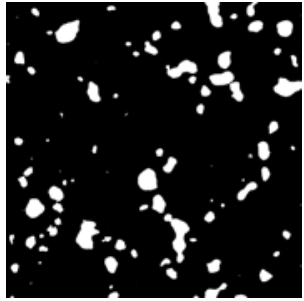

3-3

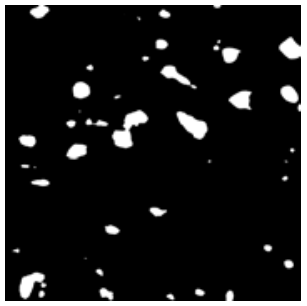

3-8

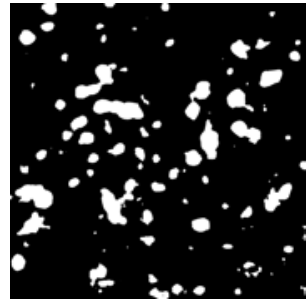

3-13

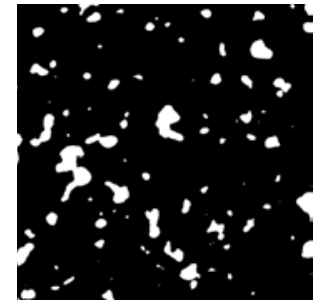

3-4

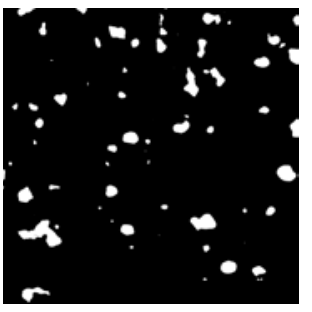

3-9

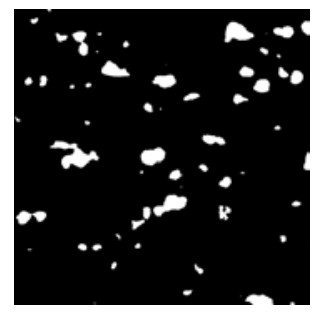

3-14

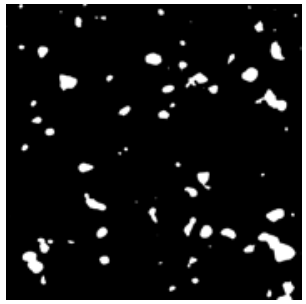

3-5

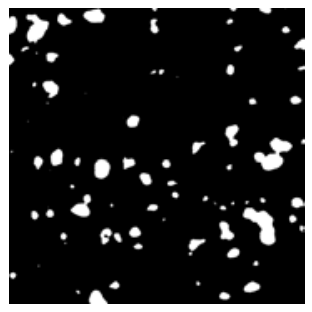

3-10

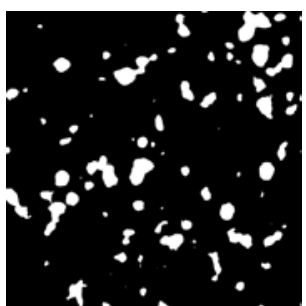

3-15

Fig. 4. Binary image of PVPP distribution of different hardness in Yinhuang dispersible tablets.

Table 1. Assemessment parameters of PVPP distrbution in Yinhuang dispersible tablets.

\begin{tabular}{|c|c|c|c|c|}
\hline Number & $\begin{array}{l}\text { Hardness } \\
(\mathrm{N})\end{array}$ & $\begin{array}{c}\text { Average } \\
\text { area } \\
\text { (pixel) }\end{array}$ & $\begin{array}{l}\text { Average } \\
\text { length } \\
\text { (pixel) }\end{array}$ & $\begin{array}{c}\text { Agglomerate } \\
\text { number }\end{array}$ \\
\hline 1 & 16 & 3972.95 & 77.4088 & 86 \\
\hline 2 & 24 & 3468.70 & 72.3987 & 95 \\
\hline 3 & 47 & 3442.27 & 72.0364 & 99 \\
\hline 4 & 52 & 3070.04 & 68.6897 & 102 \\
\hline 5 & 75 & 2686.50 & 65.8879 & 73 \\
\hline 6 & 75 & 2640.34 & 63.6160 & 78 \\
\hline 7 & 103 & 3049.09 & 66.2785 & 112 \\
\hline 8 & 111 & 4580.38 & 87.4041 & 45 \\
\hline 9 & 117 & 2908.99 & 69.1581 & 69 \\
\hline 10 & 135 & 3071.75 & 67.0281 & 84 \\
\hline 11 & 155 & 2745.23 & 62.1316 & 108 \\
\hline 12 & 158 & 3765.14 & 75.2099 & 89 \\
\hline 13 & 159 & 3984.00 & 75.4941 & 110 \\
\hline 14 & 180 & 3607.91 & 79.5383 & 62 \\
\hline 15 & 200 & 3921.38 & 79.8197 & 92 \\
\hline
\end{tabular}

\section{Conclusion}

This paper takes Yinhuang dispersible tablets as an example. PVPP distribution in Yinhuang dispersible tablets was spatially investigated using basic analysis of correlation between analysis (BACRA) method, then the BACRA analysis images were further depicted by binary image to make the spatial information of PVPP more clearly visualized. The relationship between tablet hardness and the spatial distribution uniformity of Yinhuang dispersible tablets was investigated by quantifying the agglomerate of PVPP. Finally, the best tablet hardness was obtained within $75 \mathrm{~N}$ according to the variance trend.

This work demonstrates that NIR-CI can be an effective tool in monitoring the uniformity of Yinhuang dispersible tablets, which cannot be realized by wet analysis, such as HPLC or MS. This investigation built the correlation between tablet hardness 
and the spatial distribution uniformity of Yinhuang dispersible tablets. By quantifying intra-tablet homogeneity, a new methodology to optimize the tablet hardness in CMT was proposed.

\section{Competing Interests}

The authors declare that they have no competing interests.

\section{Acknowledgments}

This work was financially supported from the National Natural Science Foundation of China (81303218), Doctoral Fund of Ministry of Education of China (20130013120006) and Innovation Team Foundation of Beijing University of Chinese Medicine, Beijing.

\section{References}

1. C. D. Ellison, B. J. Ennis, M. L. Hamad, R. C. Lyon, "Measuring the distribution of density and tabletting force in pharmaceutical tablets by chemical imaging," J. Pharm. Biomed. 48, 1-7 (2008).

2. J. M. Amigo, C. Ravn, "Direct quantification and distribution assessment of major and minor components in pharmaceutical tablets by NIR-chemical imaging," Eur. J. Pharm. Sci. 37, 76-82 (2009).

3. C. Ravn, E. Skibsted, R. Bro, "Near-infrared chemical imaging (NIR-CI) on pharmaceutical solid dosage forms-comparing common calibration approaches," J. Pharm. Biomed. 48, 554-561 (2008).

4. M. B. Lopes, J. C. Wolff, J. M. Bioucas-Dias, M. A. T. Figueiredo, "Quantification of components in non-homogenous pharmaceutical tablets using near infrared reflectance imaging," J. Near Infrared Spectrosc. 18, 333-340 (2010).

5. G. Sando, J. Dubois, "Seeing the chemicals in pharmaceutical tablets with NIR chemical imaging," Chim. Oggi 28, 40-42 (2010).

6. J. Cruz, M. Bautista, J. M. Amigo, M. Blanco, "NIR-chemical imaging study of acetylsalicylic acid in commercial tablets," Talanta 80, 473-478 (2009).

7. L. R. Hilden, C. J. Pommier, S. I. F. Badawy, E. A. Friedman, "NIR chemical imaging to guide/support
BMS-561389 tablet formulation development," Int. J. Pharm. 353, 283-290 (2008).

8. R. P. Anil, D. C. Pravin, "Novel techniques for solubility, dissolution rate and bioavailability enhancement of class II and IV drugs," Asian J. Biomed. Pharm. Sci. 2, 9-14 (2012).

9. X. P. Huang, X. X. Wan, Q. Liu, "Relationship between dissolution rate and bioavailability of paracetamol tablet," Di Yi Jun Yi Da Xue Xue Bao 23, 367-371 (2003).

10. A. S. El-Hagrasy, H. R. Morris, F. D'amico, R. A. Lodder, J. K. Drennen, "Near-Infrared spectroscopy and imaging for the monitoring of powder blend homogeneity," J. Pharm. Sci. 90, 1298-1307 (2001).

11. Y. Roggo, N. Jent, A. Edmond, "Characterizing process effects on pharmaceutical solid forms using near-infrared spectroscopy and infrared imaging," Eur. J. Pharm. Biopharm. 61, 100-110 (2005).

12. M. Saravanan, K. S. Nataraj, K. S. Ganesh, "The effect of tablet formulation and hardness on in vitro release of cephalexin from Eudragit L100 based extended release tablets," Biol. Pharm. Bull. 25, 541545 (2002).

13. Z. Wu, O. Tao, W. Cheng, L. Yu, X. Shi, Y. Qiao, "Visualizing excipient composition and homogeneity of Compound Liquorice Tablets by near-infrared chemical imaging," Spectrochim. Acta A 86, 631636 (2012).

14. Z. Wu, O. Tao, W. Cheng, L. Yun, X. Shi, Y. Qiao, "Research on spatial distribution and composition uniformity of Rukuaixiao tablets intermediate by spectral imaging," Chin J. Anal. Chem. 5, 628-634 (2011).

15. Z. Wu, O. Tao, X. Dai, M. Du, X. Shi, Y. Qiao, "Monitoring of a pharmaceutical blending process using near infrared chemical imaging," Vib. Spectrosc. 63, 371-379 (2012).

16. Y. Luo, R. Li, W. He, "Selection of excipients for Yinhuang dispersible tablets by uniform design," Acad. J. Guangdong College Pharm. 20, 117-119 (2004).

17. L. Zhou, Z. Wu, X. Shi, M. Xu, B. Xu, Y. Qiao, "Rapid discrimination of chlorpheniramine maleate and assessment of its surface content uniformity in a pharmaceutical formulation by NIR-CI coupled with statistical measurement," Spectroscopy (2014), doi:10.1155/2014/741246. 